\title{
Zebrafish as a Model to Characterize TEL2 Function During Development and Cancer
}

\author{
Anita M. Quintana and Gerard C. Grosveld*
}

Department of Genetics, St Jude Children's Research Hospital, 262 Danny Thomas Place, Memphis, TN 38105, USA

\begin{abstract}
TEL2/ETV7 is a human oncoprotein that alone or in cooperation with MYC to promote leukomogenesis. It is a member of the ETS family of transcription factors. ETS factors are common sites of chromosome translocation in cancer and are essential for normal development. Most ETS factors are conserved in mice and their function has been characterized by gain or loss of function studies. TEL2 is unique because it is not present in rodents. The lack of TEL2 in rodents has made it difficult to properly characterize the role of TEL2 in development. The genome sequencing efforts discovered that TEL2 is conserved in the zebrafish, Danio rerio, which has emerged as a powerful model system to study vasculogenesis, hematopoiesis, development, and cancer. Here, we discuss the types of experiments that have been used to characterize zebrafish ETS factors and suggest new experiments that can be performed in zebrafish to further characterize the function of TEL2.
\end{abstract}

\section{TEL2, a novel ETS transcription factor}

\section{The human TEL2 gene}

The E26 transformation specific (ETS) family of transcription factors regulates a wide variety of cellular processes including proliferation, differentiation, development, and cell death. There are approximately 30 different ETS proteins and each of them being characterized by a highly conserved DNA binding domain commonly referred to as the ETS domain. The ETS domain binds specifically to the GGAA/T consensus sequence within promoter and enhancer elements. The specificity of different ETS transcription factors is influenced by key protein interactions and by the surrounding DNA sequence. ETS proteins are subdivided into different sub-families and are highly conserved [1]. These proteins are classified on the basis of the ETS domain's location and the presence of other highly conserved domains, such as the pointed (PNT) protein-protein interaction domain [2] (Figure 1).

Since the discovery of ETS1, a plethora of highly conserved ETS proteins have been identified and characterized. Some of these proteins form fusion proteins as a result of recurrent chromosome translocations in tumors such as Ewing's sarcoma, chronic myeloid, and acute lymphoid leukemia. ETV7/TEL2, was first identified by three independent groups and was classified into the TEL subgroup (includes TEL1 and TEL2) because of its high degree of similarity to ETV6/TEL1 [3-5]. Unlike TEL1, TEL2 has a restrictive expression pattern but has in common with TEL1 that it is implicated in tumorigenesis. Here we will discuss what is known about TEL2 and compare this oncoprotein to other members of the ETS family of transcription factors. In addition, we will suggest new experiments using zebrafish (Danio rerio) as a model to characterize the role of TEL2 during normal development and tumorigenesis.

\section{Characterizing the activity of TEL2}

The TEL2 protein has a conserved ETS DNA binding domain at the C-terminus and a PNT domain at the N-terminus (Figure 1). Although TEL2 has some sequence identity to other ETS members, it is the most similar to TEL1 ( 38\%) with the highest degree of similarity in the ETS and PNT domains ( $85 \%$ and $62.5 \%$ identity, respectively) [5]. The TEL1 protein is a documented transcriptional repressor and TEL2 represses specific reporter constructs in transient transcription assays [3,5]. Although TEL2 can repress some reporter constructs in vitro, its forced expression in mice has demonstrated that TEL2 is an oncoprotein suggesting that it behaves differently in vivo. The mechanisms that convert TEL2 into an oncoprotein have remained elusive. However, a contributing factor could be that TEL1 and TEL2 form oligomers via the PNT domain. It is possible that when TEL2 is over expressed, it binds to TEL1 and prevents TEL1 from repressing transcription. These interactions are likely to occur in a tissue specific manner because TEL1 is ubiquitously expressed, but TEL2 is not.

Elucidating the role of TEL2 in cancer is complicated by the presence of TEL2 alternative transcripts. At least 6 alternative splice forms have been identified, TEL2a- TEL2f [3]. The full-length TEL2 transcript is usually referred to as TEL2b. It contains exons $1-8$. TEL2a contains an alternative exon, $3 \mathrm{a}$, which has a second start site. The subsequent transcript is bicistronic and can form a short form that consists primarily of the N-terminal region with most of the PNT domain or a second protein that starts in Exon 3 and contains the ETS domain. TEL2c has deleted exon 2 and therefore, does not have the full PNT domain, which is comprised of exons 2-4. TEL2d does not have the PNT domain due to a deletion of exon 3, but it does have the ETS domain. The TEL2e and TEL2f isoforms do not have exon 8 , which causes a truncation of the ETS domain. The primary difference between forms e and $\mathrm{f}$ is the usage of exon 3. TEL2e retains exon 3, but TEL2f deletes exon 3. Each of these variants displays different expression patterns. TEL2b is primarily found in the liver, lung, and placenta; TEL2d has a similar expression pattern. TEL2e and TEL2f are found in

*Corresponding author: Gerard Grosveld, Department of Genetics, St Jude Children's Research Hospital, 262 Danny Thomas Place, Memphis, TN 38105 , USA, Tel: +1 901595 2279; Fax: +1 901595 6035; E-mail: gerard.grosveld@stjude

Received May 30, 2011; Accepted June 22, 2011; Published June 25, 2011

Citation: Quintana AM, Grosveld GC (2011) Zebrafish as a Model to Characterize TEL2 Function During Development and Cancer. J Carcinogene Mutagene S1:001. doi:10.4172/2157-2518.S1-001

Copyright: (c) 2011 Quintana AM, et al. This is an open-access article distributed under the terms of the Creative Commons Attribution License, which permits unrestricted use, distribution, and reproduction in any medium, provided the original author and source are credited. 
the spleen and thymus, but not in the liver or lung; the other variants do not have well defined expression patterns. Most importantly, these variants appear to have different activities in reporter gene assays. The presence of these variants in cancer has not been characterized, but it is likely that these forms have a cell type specific role.

\section{TEL2 is a human oncoprotein}

To study the role of TEL2 in leukemia, researchers expressed human TEL2 in murine bone marrow progenitors and then transplanted these into lethally irradiated mice carrying the $\mathrm{E} \mu-\mathrm{Myc}$ allele, driving Myc overexpression in pre-B-cells. This translocation normally leads to preB-cell lymphoma and death in approximately 4-6 months [6,7]. The coexpression of TEL2 and E $\mu$-Myc accelerates the disease showing that the TEL2 protein cooperates with Myc overexpression during cancer formation and progression [8]. TEL2/ E-Myc mice have decreased apoptosis and increased proliferation demonstrating that TEL2 can affect cell cycle progression. These observations appear to contradict in vitro reports suggesting that TEL2 is a repressor; however it is plausible that TEL2 represses the expression of cell cycle inhibitors such as p21 and p16. Suppressing these genes could accelerate the cell cycle. Another possibility is that the in vivo role of TEL2 could be influenced by tissue specific protein interactions with TEL1, which might alter the activity of both proteins.

A subsequent study showed that forced TEL2 expression causes a myeloproliferative disorder. However, the disorder's long latency period suggests that other secondary mutations contribute to the disease [9]. Along with the studies in $\mathrm{E} \mu-\mathrm{Myc}$ mice, these data suggest that TEL2 contributes to tumorigenesis.

PNT

65-80 AA

ETS

\section{$85 \mathrm{AA}$}

ETS

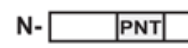

ERG

\begin{tabular}{|l|l|l|l|}
\hline & PNT & ETS & \\
\hline
\end{tabular}

ELG

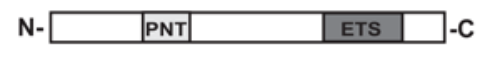

ESE

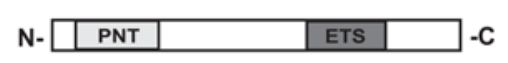

TEL

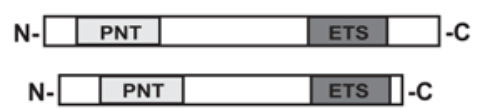

PDEF

PEA3

\section{ELF4}

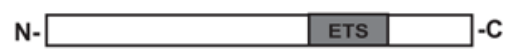

SPI

ERF

TCF

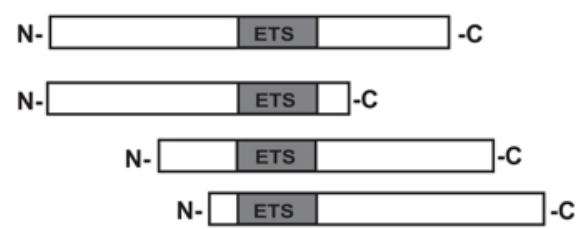

Figure 1: ETS factors are classified into distinct subclasses. The different subclasses of ETS factors are classified according to the presence or absence of the ETS domain (DNA binding domain) and the PNT domain (pointed domain). The generic structure depicted aligns each class according to the ETS domain (shaded gray) and includes the pointed domain (PNT, shaded black). The PNT domain can range from 65-80 amino acids in length and is important for proteinprotein interactions. TEL1 and TEL2 make up the TEL subclass and each has a PNT domain. These two proteins can homo and hetero dimerize. This dimerization likely affects the activity of each protein.

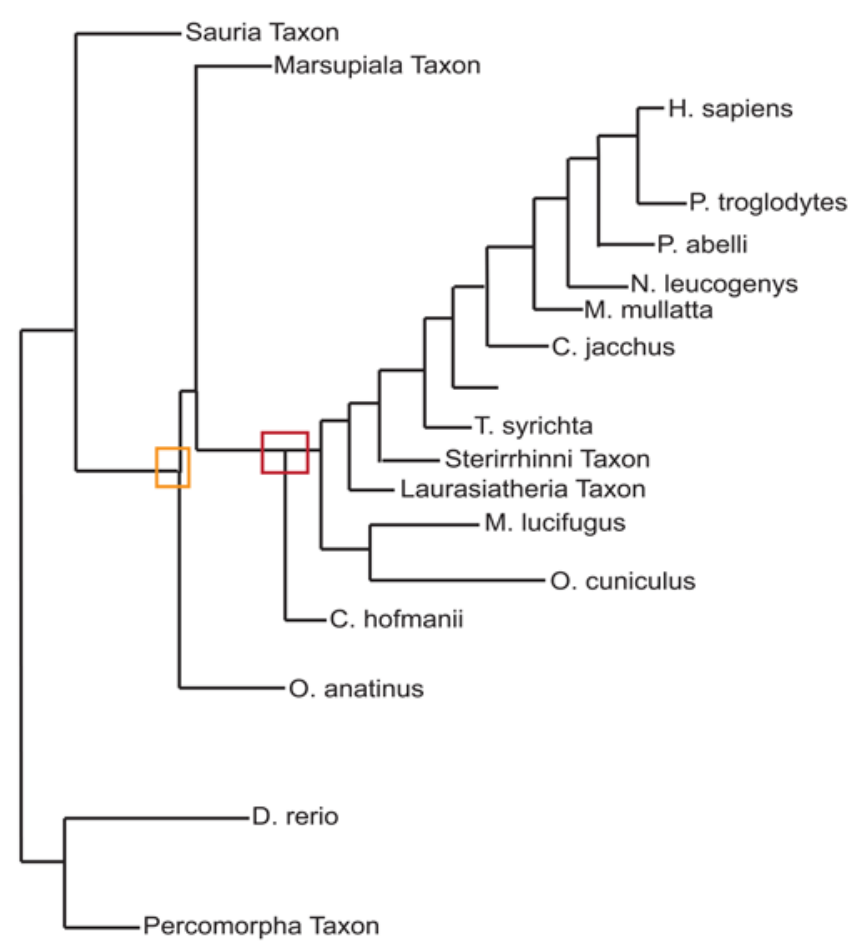

Figure 2: TEL2 is conserved in different species, but is absent from rodents. This tree depicts the species that have a TEL2 homologue. Rodents are not found on this tree, but if the gene were present in rodents it would be shown as a branch of the Murinae taxon. The Murinae taxon branches off of the Rodentia taxon, which stems from the Euarchontoglires taxon. The Euarchontoglires taxon is connected to the Eutheria taxon node, which is depicted here by the red box. All of the above taxons are part of the Mammalia taxon shown here by the orange box.

\section{A distinct role for TEL2 in tumorigenesis}

TEL1 was initially discovered as the target of a translocation in leukemia [10]. Since its initial discovery, multiple different fusion partners have been identified including PDGFRB, MN1, RUNX1 (AML1), ABL1, and JAK2, which have been reviewed elsewhere [11] (Figure 3). Other ETS proteins such as ERG, ETS1, FLI1, and PEA3 also form fusion proteins in human cancer [12,13]. These fusion proteins come in all different varieties and can include or exclude the ETS domain. Translocations excluding the ETS domain but including the PNT domain mostly create constitutively activated tyrosine kinases such as TEL1-PDGFRB, TEL1-ABL1, TEL1-JAK2 [11]. Dimerization via the PNT domain activates the linked tyrosine kinase domains of these fusion proteins, which leads to increased proliferation and inhibition of cell death $[14,15]$. An exception is TEL1/RUNX1, which contains the PNT domain from TEL1 and the DNA binding domain from RUNX1. This presumably alters the activity of RUNX1 [16]. A translocation exploiting the ETS domain of TEL1 is MN1/TEL1 [17], resulting in fusion of the transcription activation domains of MN1 to the ETS domain. This alters TEL1 from a transcriptional repressor into a transcriptional activator. Although TEL2 does not appear to be a target of chromosome translocation, its expression is frequently upregulated in human cancer (www.oncomine.org), but its role in cancer progression is unclear.

Studies in the U937 cell line have provided insight into the role of TEL2 during cancer formation and progression. These cells can be 
induced by vitamin D3 to differentiate into human monocytes. U937 cells that over-express TEL2 do not differentiate upon vitamin D3 treatment; furthermore, the amount of TEL2 mRNA decreases during differentiation suggesting that TEL2 regulates the differentiation of monocytes [18]. These data suggest that TEL2 is important for monocytic differentiation and that the forced expression of TEL2 maintains cells in an immature state.

\section{What is the normal function of TEL2?}

TEL2 is primarily expressed in the bone marrow and fetal liver [35] suggesting that it regulates hematopoiesis. As mentioned previously, TEL2 has the most sequence identity with TEL1; therefore, the function of TEL1 may provide insight into the function of TEL2. TEL1 knockout mice die by E12.5 because of defects in angiogenesis [22]. Although these mice have severe defects in vasculogenesis, hematopoeisis appears to progress normally. The role of TEL1 during vasculogenesis/ angiogenesis is similar to that of other ETS factors such as ETS1, FLI1, and ERG. All of these proteins are expressed in endothelial cells and are necessary for proper angiogenesis/vasculogenesis [21].

It has been difficult to characterize the role of TEL1 during development because TEL1 deficient mice die in utero. The use of chimeric mice circumvented this problem because chimeric mice are viable and can be engineered to express blood cells that exhibit gain or loss of function alleles. TEL1-/- chimeras have decreased numbers of B and T-cells in the bone marrow, but have sufficient amounts of B and T-cells in the secondary lymphoid organs (i.e. spleen and thymus) [23]. These data suggest that TEL1 regulates the number of $B$ and $\mathrm{T}$-cells in the bone marrow and contributes to proper regulation of hematopoiesis.

TEL2 is present in vertebrates but has been deleted in rodents while the closely related lagomorphs maintained the gene (Ensembl.org). (Figure 2) represents a phylogenetic tree of species that retain TEL2. Rodents are not listed because the gene has been deleted. Because of the deletion of TEL2, we hypothesize that the function of the TEL2 gene will be better characterized in another species. The zebrafish genome sequencing effort confirmed the existence of TEL2 in zebrafish by performing sequence alignment with the human TEL2 gene and the annotated zebrafish TEL2 (zTEL2). zTEL2 is $49 \%$ homologous with human TEL2 with a high degree of similarity in the ETS and PNT domains (85\% and 73\% identity, respectively) (Figure 3). zTEL2 also shares some degree of homology with the human TEL1 gene, where the ETS domain has $86 \%$ identity, but the PNT domain has 56\% identity. The presence of this highly conserved ortholog in zebrafish suggests that zebrafish can be used as a model to characterize zTEL2 function during normal development. Zebrafish are an excellent model system because of its external fertilization, translucency, and high fecundity.

\section{Vertebrate Hematopoiesis}

Many of the mechanisms regulating vertebrate hematopoiesis have been reviewed elsewhere [24,25]; however because TEL2 may play a significant role regulating hematopoiesis, we will briefly compare and contrast the process of hematopoieisis in zebrafish with that of higher vertebrates. All vertebrate hematopoiesis occurs in two waves. In the first wave (primitive hematopoiesis), red blood cells and macrophages are produced in the extraembryonic yolk sac. These initial cells are required for growth of the organism, but are not multipotent. The second wave (definitive hematopoiesis) occurs in the aortagonad-mesonephros (AGM) region and results in the production of multipotent stem cells that form multiple blood cell lineages.

Zebrafish hematopoiesis occurs in two stages, but both stages occur intraembryonically. Primitive hematopoiesis occurs at two different sites in the developing embryo. Hematopoiesis begins in the ventral mesoderm (that is formed from inner cell mass), but the production of red blood cells and myeloid cells is segregated within the embryo. Red blood cells are primarily produced in the embryo's posterior end whereas myeloid cells are produced in the embryo's anterior end. Definitive hematopoiesis occurs primarily in the caudal hematopoietic tissue (CHT) and eventually, adult stem cells migrate to secondary tissues such as the thymus and kidney. Because of the

\section{ETS Domain Homology}

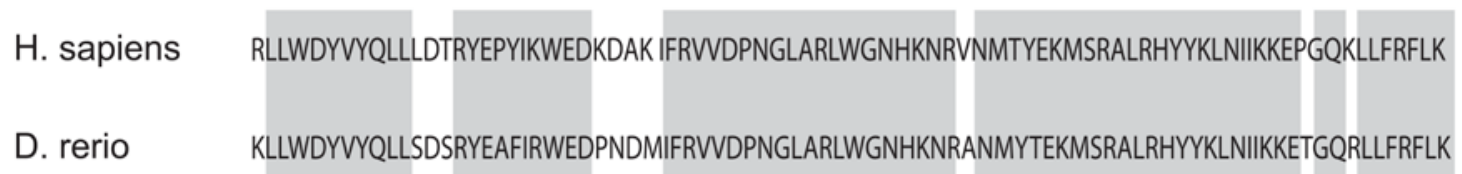

PNT Domain Homology

H. sapiens NLLGEGGICKLPGRLRI QPALWSREDVLHWLRWAEQEYSLPCTAEHG FEMNGR ALCI LTKDDFRHRAP SGDVLYELLQYIKT QRRAL

D. rerio

Figure 3: Alignment of human and zebrafish TEL2 functional domains. Top: The zebrafish TEL2 ETS domain is approximately $85 \%$ identical to the human TEL2 ETS domain. The regions of similarity are shown in gray. Bottom: The PNT domains from human TEL2 and zebrafish TEL2 are shown. They exhibit a high degree of sequence identity, but less than the ETS domain. Regions of similarity are shown in gray. 
strikingly conserved mechanisms of higher vertebrate and zebrafish hematopoiesis, it is likely that zebrafish can be used to determine the role of TEL2 during hematopoiesis.

\section{The role of ETS factors during zebrafish development \\ Using zebrafish to our advantage}

Many groups have started using zebrafish to study the role of ETS factors in vertebrate hematopoiesis. Gain or loss of function assays can be performed quickly in zebrafish by microinjecting antisense oligonucleotides or transiently expressing in vitro synthesized mRNA. The primary advantage of using zebrafish for these types of studies is the ability to visualize morphological changes early in the developmental timeline. In some cases, transgenic zebrafish expressing fluorescently tagged hematopoietic markers have been used to analyze the effects of gene deletion or over expression. These experiments, combined with whole mount in situ hybridization can pinpoint both the expression and the biological function of ETS genes during this process.

\section{ETS factors that regulate hematopoiesis}

The SPI family of ETS factors consists of PU.1/SPI, SPIB, and SPIC. In zebrafish two homologous SPI factors have been identified, PU.1 and SPI-11. In humans, PU.1 is expressed in hematopoietic cells and controls proper differentiation of myeloid and lymphoid cells [26,27]. Deletion of PU.1 is lethal in mice [28,29], but since zebrafish are externally fertilized and hematopoietic mechanisms are highly conserved in vertebrates some groups have used zebrafish to characterize PU.1 function.

PU.1 expression begins at the 12-somite stage of zebrafish development and is localized in the anterior lateral mesoderm [30]. Approximately 16 hours after fertilization, PU.1 expression can be detected in both the anterior and posterior regions of the animal and by 22 hours post fertilization (hpf) expression in the posterior region is lost [31]. PU.1 expression overlaps with Scl, a myeloid specific marker [30], but not with Gata1 [32] suggesting that PU.1 is essential for primitive myelopoiesis, but not erythropoeisis. In fact, when PU.1 is absent, the differentiation of cells within the anterior region of the animal is skewed towards the red blood cell lineage [33]. These results have been confirmed with an enhanced green fluorescent protein (EGFP) reporter gene in which EGFP expression is regulated by elements of the PU.1 promoter [34]. Although previous work had not demonstrated a role for PU.1 in adult hematopoiesis, EGFP expression was found in a small subset of cells that were MPO positive and a subset of lymphoid progenitors within the kidney, the site of adult hematopoiesis.

$S p i-1 l$ is a novel zebrafish ETS protein with a high degree of similarity to the human and zebrafish PU.1. Spi-1l is expressed in the anterior lateral plate mesoderm as early as the 6-somite stage suggesting that it regulates hematopoiesis [35]. Spi-1l knockdown alters the expression of MPO and is up regulated by exogenous expression of PU.1. These data suggest that $P U .1$ and Spi-1l share some overlapping functions and but also have some unique functions during hematopoiesis.

\section{Vertebrate Vasculogenesis/Angiogenesis}

Vasculogenesis occurs as a multi-step process. The vasculature is among the first organs produced during development. The process begins with the differentiation of hemangioblasts from the extraembryonic mesodermal cells. Hemangioblasts aggregate and form blood islands in the yolk sac and then differentiate to form an external endothelial cell layer and an internal blood layer. This structure is referred to as the primary vascular plexus. Similarly, intraembryonic hemangioblasts derived from the lateral mesoderm differentiate into endothelial cells that form the dorsal aorta. After these two structures are formed, new vessels are distributed throughout the rest of the body. The original formation of the vasculature is called vasculogenesis; the subsequent extension is referred to as angiogenesis [36].

Vasculogenesis in zebrafish is very similar to that in higher vertebrates. The endothelial cells are derived from hemangioblast precursors. Hemangioblasts are visible in the lateral plate mesoderm $12 \mathrm{hpf}$ and differentiate into endothelial cells by $17 \mathrm{hpf}$ [37]. By 20 hpf zebrafish have formed the dorsal aorta and the posterior cardinal vein. Formation of new blood vessels occurs between 20-24 hpf and by $30 \mathrm{hpf}$ vessels are connected to the dorsal aorta. Zebrafish are unique because they can survive up to a week without a vasculature [37], while most other animal models succumb to death almost immediately. One example of this phenomenon is the zebrafish cloche mutant, which does not develop blood cells but has been used in numerous studies up to $26 \mathrm{hpf}[38,39]$.

\section{ETS factors regulate angiogenesis in zebrafish}

There are three zebrafish ETS factors that have been shown to regulate angiogenesis, FLI1, etsrp, and erg. FLI1 was originally identified as the Friend murine leukemia virus' target site of integration [40] and is expressed in hematopoietic tissues in the mouse [41]. In zebrafish FLI1 is expressed as early as $10 \mathrm{hpf}$ and by $19-22 \mathrm{hpf}$ is found in regions that give rise to endothelial cells and hematopoietic precursors [42]. As development progresses, FLI1 is localized to the zebrafish brain and within precursors of the heart endothelium. Knockdown of FLI1 results in severe brain hemorrhage at $72 \mathrm{hpf} \mathrm{[38]} \mathrm{and} \mathrm{mutant} \mathrm{zebrafish}$ that do not form a proper vasculature have decreased FLI1 expression.

Three independent groups identified etsrp as a potent regulator of vasculogenesis $[38,43,44]$. The expression of etsrp is very similar to that of FLI1 etsrp-knockdown has been shown to cause a complete loss of circulation [44]. Furthermore, an etsrp mutant, y11, has a beating heart, but does not have circulation in its trunk [43]. These effects may be mediated through the induction of genes such as $s c l$ and $f l k 1$ $[38,44]$. Interestingly, etsrp does not alter gatal expression, which marks hemangioblasts that give rise to erythrocytes and endothelial cells suggesting that only the subset of hemangioblasts that give rise to myeloid and endothelial cells are affected by etsrp knockdown. These data provide implications for spatial differences in the differentiation program of early progenitors and suggest that environmental factors influence differentiation.

Erg (ETS related gene) was originally identified using microarray analysis [45] and is expressed in the vasculature of the brain, trunk, and tail [46]. Over expressing erg protein increases angioblast proliferation suggesting that $\operatorname{erg}$ regulates the proliferation of immature precursors. However, erg is not essential for this process because erg knockdown does not affect vascular development [46]. These data suggest that other factors may play a redundant role during vasculogenesis. It is plausible that either etsrp or FLI1 mediates this redundancy, given that their expression patterns are relatively similar.

Does TEL2 regulate hematopoiesis, vasculogenesis, or angiogenesis?

As discussed previously, numerous different ETS factors are expressed during zebrafish development and play critical roles in the development of proper hematopoiesis and vasculogenesis/ 
angiogenesis. Little is known about the function of TEL2 during these processes, however, since other ETS factors have overlapping functions it is plausible that TEL2 has a role in these developmental processes (Figure 4). Because zebrafish have an evolutionarily conserved TEL2 gene, we propose using zebrafish to determine what role (if any) TEL2 might play during hematopoiesis, vasculogenesis, and angiogenesis.

\section{Are zebrafish a good model to study cancer?}

Zebrafish have been used for decades to study the mechanisms and signaling cascades regulating proper development. In recent years, many researchers have realized that cancer cells have the capability to highjack normal developmental pathways such as the WNT or sonic hedgehog pathways to accelerate proliferation and escape cell death $[47,48]$. These pathways are highly conserved from zebrafish to humans, a fact which prompted scientists to use zebrafish as a model of carcinogenesis. Furthermore, zebrafish tumors are very similar to tumors in mice and humans, making zebrafish a good model to study how developmental pathways go awry in cancer [49].

Recently, three independent studies were performed that validate the efficacy of zebrafish as model for carcinogenesis. In each case a fusion protein derived from chromosomal translocation was expressed in zebrafish embryos. These fusion proteins were originally identified in humans and have been studied extensively in mice. These studies demonstrate that zebrafish develop cancers that are similar in pathology to human cancers and suggest that future work characterizing the mechanisms causing cancer should include zebrafish.

\section{AML1: ETO}

AML1:ETO was originally identified in acute myeloid leukemia [50]. The translocation results in the fusion of AML1 (RUNX1) with ETO (CBF2T1). In the absence of AML1, mice develop hemorrhages in the central nervous system and have defects in hematopoiesis [51,52]; furthermore, over expression of AML1: ETO in mice causes death in utero [53]. Recently, these data have been extended in a zebrafish model of leukemia, which expresses the human AML1-ETO protein [54]. The transient overexpression of AML1:ETO caused blood cell defects and central nervous system hemorrhaging [54]. This phenotype is similar to

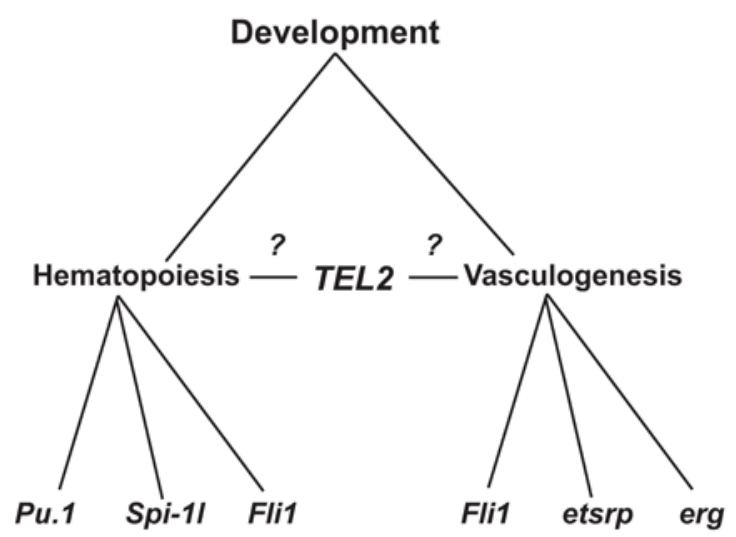

Figure 4: ETS factors are important for normal development. Different classes of ETS transcription factors regulate different biological processes. The SPI class specifically regulates normal hematopoiesis, while other ETS proteins such as FLI1 are essential for vasculogenesis and angiogenesis. TEL2 might regulate both hematopoiesis and vasculogenesis. In this review we suggest that zebrafish are a powerful model system to study TEL2 function. the phenotype observed following AML1 knockdown [54] suggesting that fusing ETO to AML1 acts as a dominant negative mutation. This phenomenon has also been observed in mice expressing AML1:ETO [55].

\section{TEL-JAK2a}

TEL-Jak2a is a fusion that was identified in cancer. It fused the PNT domain of TEL1 with the kinase domain of Jak2 making a constitutively activated kinase [56]. Expressing exogenous TEL1:Jak2a in mice causes T-cell leukemia [57], which suggests that this fusion protein is a potent oncoprotein. For these reasons, TEL1:Jak2a was transiently expressed in zebrafish. Over-expression caused defects in normal blood circulation where red blood cells do not contain hemoglobin and accumulate near the animal's heart [58]. Furthermore, injected animals had more white blood cells relative to red blood cells and the expression of Spi-1l, a marker of myeloid differentiation, is elevated in the injected animals [58]. Taken together, these data demonstrate that TEL1: Jak2a alters the differentiation program and causes up regulation of white blood cells at the expense of erythropoiesis, which could contribute to leukemia formation.

\section{TEL1:AML1}

The TEL1:AML1 fusion protein is present in approximately $25 \%$ of all childhood pre-B-cell acute lymphoblastic leukemia and is the most common translocation in childhood leukemia [59]. TEL1: AML1 is not highly oncogenic when over expressed in mouse models, but can be highly tumorigenic in the presence of other synergistic mutations [60,61]. In addition TEL1: AML1 can be present during normal fetal development, but only specific subsets of individuals develop leukemia [62].

Zebrafish engineered to express TEL1: AML1 under the control of a $\beta$-actin (ubiquitous) promoter developed hyperplasia and subsequent leukemia [63]. The leukemia observed in zebrafish exhibits down regulation of endogenous TEL1, which has been observed in human cancers that express TEL1: AML1 [64-66]. The leukemias also have deregulation of genes that normally regulate cell cycle and apoptosis [63]. These data suggest that TEL1: AML1 tumorigenesis depends on the presence of other cooperating mutations.

\section{Characterizing TEL2 function in zebrafish}

We discussed three independent studies characterizing the role of fusion proteins during carcinogenesis in zebrafish. All three studies provide evidence that zebrafish are a potent model of cancer and provide a foundation for future research in this area. For the remaining portion of this review, we will discuss potential experiments that can be used to characterize TEL2 during normal development and cancer. TEL2 is one of the few ETS transcription factors that is conserved in vertebrates, but not in mice; however, forced TEL2 protein expression can drive tumorigenesis [9]. The primary mechanism that ETS transcription factors use to contribute to tumorigenesis is chromosomal translocation, but to date there has been no report of chromosomal translocation involving TEL2 in human leukemia. The fact that TEL2 expression is upregulated in many human tumors (www.oncomine.org) suggests that TEL2 may play an important but poorly understood role in cancer development. Because TEL2 is not expressed in rodents, it becomes necessary to use alternative models to characterize the function of TEL2 in cancer. Given that zebrafish TEL2 (zTEL2) is highly conserved suggests that this is a promising model 
system by which to determine the normal and tumorigenic role of the zTEL2.

\section{The feasibility of gain or loss of function assays}

Zebrafish biologists have used a variety of different mechanisms to change the expression of target genes. Transient overexpression studies can be performed by injection of in vitro transcribed mRNA. This can lead to expression of a particular gene throughout the organism. A more advantageous means of over expression is the creation of transgenic zebrafish. In recent years, it has become possible to efficiently make transgenic fish that over express a particular gene under tissue specific promoters. In the past, transgenic zebrafish were produced primarily by the injection of plasmid DNA. However, the efficiency of this method was relatively low (approximately 1-10\%) [67]. Transduction efficiency has increased with the advent of the Tol2kit, a multisite gateway cloning kit [68]. Multiple groups have demonstrated that this method is more efficient and can be used to produce inducible transgenes $[68,69]$.

Loss of function studies can be performed with a variety of different tools. One of the most popular means of knocking down a gene is to use morpholino oligos to inhibit translation or alternative splicing. Morpholinos are antisense oligonucleotides that have a morpholino ring in place of the ribose ring that is normally present in DNA and RNA [70]. This method is relatively fast and cheap. Other methods like Zinc-Finger nucleases (ZFNs) have emerged as a powerful tool to produce stable transgenic lines with site-specific genetic alteration. ZFNs are fusion proteins that contain a zinc-finger protein fused to the cleavage domain of FokI endonuclease [71]. These engineered proteins can be designed to cleave specific DNA sequences $[71,72]$ and the introduction of ZFNs at the one cell stage has led to stable heritable transmission of mutant alleles in zebrafish [73,74]. These two methods include the injection of DNA oligonucleotides or the laborious design of proteins that specifically recognize a specific DNA sequence. Other methods such as TILLING (Targeting Induced Local Lesions in Genomes) are efficient means to construct null transgenic lines. TILLING is a method based on random mutagenesis and has been used to generate transgenic lines of fish with mutations in p53 and TSC2 $[75,76]$.

\section{Antibodies: The missing reagent?}

Research with zebrafish has advanced immensely in recent years. Although zebrafish biology includes powerful techniques such as in situ hybridization, tissue sectioning/pathology, morpholinos, and the Tol2kit; other useful tools are still lacking. For example, antibodies that recognize zebrafish homologues are not routinely produced. Antibodies allow researchers to study protein: protein interactions, protein: DNA interactions, localization, and post-translational modifications. zTEL2 is a transcription factor that regulates gene expression. It is likely to interact with other proteins and it might be modified by posttranslational modifications. These interactions and modifications will affect zTEL2 activity and determine the specificity of zTEL2 when it interacts with DNA. Therefore, in order to more accurately characterize zTEL2 function, antibodies that recognize zTEL2 will be essential.

\section{Concluding Remarks}

ETS transcription factors are linked to a variety of different types of cancer. A recent member of the ETS family, TEL2, can drive tumorigenisis and cooperate with Myc to accelerate leukemic progression in mice [8]. A number of other ETS factors are involved in translocations that affect cancer formation. Although TEL2 has not been shown to be a target of chromosomal translocation its expression is frequently upregulated in human cancer. TEL2 is not found in rodents, which has hindered our efforts to characterize TEL2's normal function during embryonic development. Because the mechanisms regulating proliferation, hematopoiesis, and angiogenesis are very similar in humans and zebrafish, we believe that studying $z$ TEL2 will help elucidate the role of TEL2 in human development and provide novel insights in human cancer.

\section{References}

1. Degnan BM, Degnan SM, Naganuma T, Morse DE (1993) The ets multigene family is conserved throughout the Metazoa. Nucleic Acids Res 21: 3479-84.

2. Sato Y (2001) Role of ETS family transcription factors in vascular development and angiogenesis. Cell Struct Funct 26: 19-24.

3. Gu X, Shin BH, Akbarali Y, Weiss A, Boltax J, et al. (2001) Tel-2 is a nove transcriptional repressor related to the Ets factor Tel/ETV-6. J Biol Chem 276: 9421-9436.

4. Poirel H, Lopez RG, Lacronique V, Della Valle V, Mauchauffé M, et al. (2000) Characterization of a novel ETS gene, TELB, encoding a protein structurally and functionally related to TEL. Oncogene 19: 4802-4806.

5. Potter MD, Buijs A, Kreider B, van Rompaey L, Grosveld GC (2000) Identification and characterization of a new human ETS-family transcription factor, TEL2, that is expressed in hematopoietic tissues and can associate with TEL1/ETV6. Blood 95: 3341-3348.

6. Hayday AC, Gillies SD, Saito H, Wood C, Wiman K, et al. (1984) Activation of a translocated human c-myc gene by an enhancer in the immunoglobulin heavychain locus. Nature 307: 334-340.

7. Adams JM, Harris AW, Pinkert CA, Corcoran LM, Alexander WS, et al. (1985) The c-myc oncogene driven by immunoglobulin enhancers induces lymphoid malignancy in transgenic mice. Nature 318: 533-538

8. Cardone M, Kandilci A, Carella C, Nilsson JA, Brennan JA, et al. (2005) The novel ETS factor TEL2 cooperates with Myc in B lymphomagenesis. Mol Cell Biol 25: 2395-2405.

9. Carella C, Potter M, Bonten J, Rehg JE, Neale G, et al. (2006) The ETS factor TEL2 is a hematopoietic oncoprotein. Blood 107: 1124-1132.

10. Golub TR, Barker GF, Lovett M, Gilliland DG (1994) Fusion of PDGF receptor beta to a novel ets-like gene, tel, in chronic myelomonocytic leukemia with $\mathrm{t}(5 ; 12)$ chromosomal translocation. Cell $77: 307-316$.

11. Bohlander SK (2005) ETV6: a versatile player in leukemogenesis. Semin Cancer Biol 15: 162-174.

12. Janknecht R (2005) EWS-ETS oncoproteins: the linchpins of Ewing tumors Gene 363: 1-14.

13. Seth A, Watson DK (2005) ETS transcription factors and their emerging roles in human cancer. Eur J Cancer 41: 2462-2478.

14. Kelly LM, Gilliland DG (2002) Genetics of myeloid leukemias. Annu Rev Genomics Hum Genet 3: 179-198.

15. Dash A, Gilliland DG (2001) Molecular genetics of acute myeloid leukaemia Best Pract Res Clin Haematol 14: 49-64.

16. Golub TR, Barker GF, Bohlander SK, Hiebert SW, Ward DC, et al. (1995) Fusion of the TEL gene on 12p13 to the AML1 gene on 21q22 in acute lymphoblastic leukemia. Proc Natl Acad Sci U S A 92: 4917-4921.

17. Buijs A, van Rompaey L, Molijn AC, Davis JN, Vertegaal AC, et al. (2000) The MN1-TEL fusion protein, encoded by the translocation $(12 ; 22)(\mathrm{p} 13 ; \mathrm{q} 11)$ in myeloid leukemia, is a transcription factor with transforming activity. Mol Cel Biol 20: 9281-93.

18. Kawagoe H, Potter M, Ellis J, Grosveld GC (2004) TEL2, an ETS facto expressed in human leukemia, regulates monocytic differentiation of U937 Cells and blocks the inhibitory effect of TEL1 on ras-induced cellular transformation. Cancer Res 64: 6091-6100. 
19. Behre G, Zhang P, Zhang DE, Tenen DG (1999) Analysis of the modulation of transcriptional activity in myelopoiesis and leukemogenesis. Methods 17: 231237

20. Koschmieder S, Rosenbauer F, Steidl U, Owens BM, Tenen DG (2005) Role of transcription factors C/EBPalpha and PU.1 in normal hematopoiesis and leukemia. Int J Hematol 81: 368-377.

21. Maroulakou IG, Bowe DB (2000) Expression and function of Ets transcription factors in mammalian development: a regulatory network. Oncogene 19: 643242.

22. Wang LC, Kuo F, Fujiwara Y, Gilliland DG, Golub TR, et al. (1997) Yolk sac angiogenic defect and intra-embryonic apoptosis in mice lacking the Ets-related factor TEL. EMBO J 16: 4374-4383.

23. Wang LC, Swat W, Fujiwara Y, Davidson L, Visvader J, et al. (1998) The TEL ETV6 gene is required specifically for hematopoiesis in the bone marrow. Genes Dev 12: 2392-2402.

24. Ellett F, Lieschke GJ (2010) Zebrafish as a model for vertebrate hematopoiesis. Curr Opin Pharmacol 10: 563-570.

25. Paik EJ, Zon LI (2010) Hematopoietic development in the zebrafish. Int J Dev Biol 54: 1127-1137.

26. Hromas R, Orazi A, Neiman RS, Maki R, Van Beveran C, et al. (1993) Hematopoietic lineage- and stage-restricted expression of the ETS oncogene family member PU.1. Blood 82: 2998-3004.

27. Tenen DG, Hromas R, Licht JD, Zhang DE (1997) Transcription factors, normal myeloid development, and leukemia. Blood 90: 489-519.

28. McKercher SR, Torbett BE, Anderson KL, Henkel GW, Vestal DJ, et al (1996) Targeted disruption of the PU.1 gene results in multiple hematopoietic abnormalities. EMBO J 15: 5647-5658.

29. Scott EW, Simon MC, Anastasi J, Singh H (1994) Requirement of transcription factor PU.1 in the development of multiple hematopoietic lineages. Science 265: 1573-1577.

30. Lieschke GJ, Oates AC, Paw BH, Thompson MA, Hall NE, et al. (2002) Zebrafish SPI-1 (PU.1) marks a site of myeloid development independent of primitive erythropoiesis: implications for axial patterning. Dev Biol 246: $274-$ 295.

31. Bennett CM, Kanki JP, Rhodes J, Liu TX, Paw BH, et al (2001) Myelopoiesis in the zebrafish, Danio rerio. Blood 98: 643-651.

32. Galloway JL, Wingert RA, Thisse C, Thisse B, Zon LI (2005) Loss of gata1 but not gata2 converts erythropoiesis to myelopoiesis in zebrafish embryos. Dev Cell 8: 109-116.

33. Rhodes J, Hagen A, Hsu K, Deng M, Liu TX, et al. (2005) Interplay of pu.1 and gata1 determines myelo-erythroid progenitor cell fate in zebrafish. Dev Cell 8: 97-108.

34. Hsu K, Traver D, Kutok JL, Hagen A, Liu TX, et al. (2004) The pu.1 promoter drives myeloid gene expression in zebrafish. Blood 104: 1291-1297.

35. Bukrinsky A, Griffin KJ, Zhao Y, Lin S, Banerjee U (2009) Essential role of spi1-like (spi-1l) in zebrafish myeloid cell differentiation. Blood 113: 2038-46.

36. Lelièvre E, Lionneton F, Soncin F, Vandenbunder B (2001) The Ets family contains transcriptional activators and repressors involved in angiogenesis. Int J Biochem Cell Biol 33: 391-407.

37. Baldessari D, Mione M (2008) How to create the vascular tree? (Latest) help from the zebrafish. Pharmacol Ther 118: 206-230.

38. Liu F, Patient R (2008) Genome-wide analysis of the zebrafish ETS family identifies three genes required for hemangioblast differentiation or angiogenesis. Circ Res 103: 1147-1154.

39. Stainier DY, Weinstein BM, Detrich HW 3rd, Zon LI, Fishman MC (1995) Cloche, an early acting zebrafish gene, is required by both the endothelial and hematopoietic lineages. Development 121: 3141-3150.

40. Ben-David Y, Giddens EB, Letwin K, Bernstein A (1991) Erythroleukemia induction by Friend murine leukemia virus: insertional activation of a new member of the ets gene family, Fli-1, closely linked to c-ets-1. Genes Dev 5 : 908-18.
41. Mélet F, Motro B, Rossi DJ, Zhang L, Bernstein A (1996) Generation of a novel Fli-1 protein by gene targeting leads to a defect in thymus development and a delay in Friend virus-induced erythroleukemia. Mol Cell Biol 16: 2708-2718.

42. Brown LA, Rodaway AR, Schilling TF, Jowett T, Ingham PW, et al. (2000) Insights into early vasculogenesis revealed by expression of the ETS-domain transcription factor Fli-1 in wild-type and mutant zebrafish embryos. Mech Dev 90: $237-252$

43. Pham VN, Lawson ND, Mugford JW, Dye L, Castranova D, et al. (2007) Combinatorial function of ETS transcription factors in the developing vasculature. Dev Biol 303: 772-783.

44. Sumanas S, Lin S (2006) Ets1-related protein is a key regulator of vasculogenesis in zebrafish. PLoS Biol 4: e10.

45. Weber GJ, Choe SE, Dooley KA, Paffett-Lugassy NN, Zhou Y, et al. (2005) Mutant-specific gene programs in the zebrafish. Blood 106: 521-530.

46. Ellett F, Kile BT, Lieschke GJ (2009) The role of the ETS factor erg in zebrafish vasculogenesis. Mech Dev 126: 220-229.

47. Sugimura R, Li L (2010) Noncanonical Wnt signaling in vertebrate development, stem cells, and diseases. Birth Defects Res C Embryo Today 90: 243-256.

48. Chari NS, McDonnell TJ (2007) The sonic hedgehog signaling network in development and neoplasia. Adv Anat Pathol 14: 344-352.

49. Amatruda JF, Patton EE (2008) Genetic models of cancer in zebrafish. Int Rev Cell Mol Biol 271: 1-34.

50. Bitter MA, Le Beau MM, Rowley JD, Larson RA, Golomb HM, et al. (1987) Associations between morphology, karyotype, and clinical features in myeloid leukemias. Hum Pathol 18: 211-225.

51. Okuda T, van Deursen J, Hiebert SW, Grosveld G, Downing JR (1996) AML1, the target of multiple chromosomal translocations in human leukemia, is essential for normal fetal liver hematopoiesis. Cell 84: 321-30.

52. Wang Q, Stacy T, Binder M, Marin-Padilla M, Sharpe AH, et al. (1996) Disruption of the Cbfa2 gene causes necrosis and hemorrhaging in the centra nervous system and blocks definitive hematopoiesis. Proc Natl Acad Sci U S A 93: $3444-3449$

53. Yergeau DA, Hetherington CJ, Wang Q, Zhang P, Sharpe AH, et al. (1997) Embryonic lethality and impairment of haematopoiesis in mice heterozygous for an AML1-ETO fusion gene. Nat Genet 15: 303-306.

54. Kalev-Zylinska ML, Horsfield JA, Flores MV, Postlethwait JH, Vitas MR, et al. (2002) Runx1 is required for zebrafish blood and vessel development and expression of a human RUNX1-CBF2T1 transgene advances a model for studies of leukemogenesis. Development 129: 2015-2030.

55. Sun, W. and J.R. Downing (2004) Haploinsufficiency of AML1 results in a decrease in the number of LTR-HSCs while simultaneously inducing an increase in more mature progenitors. Blood 104: 3565-3572.

56. Lacronique V, Boureux A, Valle VD, Poirel H, Quang CT, et al. (1997) A TEL-JAK2 fusion protein with constitutive kinase activity in human leukemia Science 278: 1309-1312

57. Carron C, Cormier F, Janin A, Lacronique V, Giovannini M, et al. (2000) TEL JAK2 transgenic mice develop T-cell leukemia. Blood 95: 3891-3899.

58. Onnebo SM, Condron MM, McPhee DO, Lieschke GJ, Ward AC (2005) Hematopoietic perturbation in zebrafish expressing a tel-jak2a fusion. Exp Hematol 33: 182-188.

59. Pui CH, Relling MV, Downing JR (2004) Acute lymphoblastic leukemia. N Eng J Med 350: 1535-1548

60. Andreasson P, Schwaller J, Anastasiadou E, Aster J, Gilliland DG (2001) The expression of ETV6/CBFA2 (TEL/AML1) is not sufficient for the transformation of hematopoietic cell lines in vitro or the induction of hematologic disease in vivo. Cancer Genet Cytogenet 130: 93-104.

61. Bernardin F, Yang Y, Cleaves R, Zahurak M, Cheng L, et al. (2002) TEL-AML1, expressed from $\mathrm{t}(12 ; 21)$ in human acute lymphocytic leukemia, induces acute leukemia in mice. Cancer Res 62: 3904-3908.

62. Mori H, Colman SM, Xiao Z, Ford AM, Healy LE, et al. (2002) Chromosome 
Citation: Quintana AM, Grosveld GC (2011) Zebrafish as a Model to Characterize TEL2 Function During Development and Cancer. J Carcinogene Mutagene S1:001. doi:10.4172/2157-2518.S1-001

Page 8 of 8

translocations and covert leukemic clones are generated during normal fetal development. Proc Natl Acad Sci U S A 99: 8242-8247.

63. Sabaawy HE, Azuma M, Embree LJ, Tsai HJ, Starost MF, et al. (2006) TELAML1 transgenic zebrafish model of precursor B cell acute lymphoblastic leukemia. Proc Natl Acad Sci U S A 103: 15166-15171.

64. Romana SP, Poirel H, Leconiat M, Flexor MA, Mauchauffé M, et al. (1995) High frequency of $t(12 ; 21)$ in childhood B-lineage acute lymphoblastic leukemia. Blood 86: 4263-4269.

65. Romana SP, Mauchauffé M, Le Coniat M, Chumakov I, Le Paslier D, et al. (1995) The $t(12 ; 21)$ of acute lymphoblastic leukemia results in a tel-AML1 gene fusion. Blood 85: 3662-3670.

66. Patel N, Goff LK, Clark T, Ford AM, Foot N, et al. (2003) Expression profile of wild-type ETV6 in childhood acute leukaemia. Br J Haematol 122: 94-98.

67. Stuart GW, McMurray JV, Westerfield M (1988) Replication, integration and stable germ-line transmission of foreign sequences injected into early zebrafish embryos. Development 103: 403-412.

68. Kwan KM, Fujimoto E, Grabher C, Mangum BD, Hardy ME, et al. (2007) The Tol2kit: a multisite gateway-based construction kit for Tol2 transposon transgenesis constructs. Dev Dyn 236: 3088-3099.

69. Hans S, Kaslin J, Freudenreich D, Brand M (2009) Temporally-controlled site- specific recombination in zebrafish. PLoS One 4: e4640.

70. Eisen JS, Smith JC (2008) Controlling morpholino experiments: don't stop making antisense. Development 135: 1735-1743.

71. Kim YG, Cha J, Chandrasegaran S (1996) Hybrid restriction enzymes: zinc finger fusions to Fok I cleavage domain. Proc Natl Acad Sci U S A 93: 11561160 .

72. Carroll D, Morton JJ, Beumer KJ, Segal DJ (2006) Design, construction and in vitro testing of zinc finger nucleases. Nat Protoc 1: 1329-1341.

73. Meng X, Noyes MB, Zhu LJ, Lawson ND, Wolfe SA (2008) Targeted gene inactivation in zebrafish using engineered zinc-finger nucleases. Nat Biotechno 26: 695-701.

74. Doyon Y, McCammon JM, Miller JC, Faraji F, Ngo C, et al. (2008) Heritable targeted gene disruption in zebrafish using designed zinc-finger nucleases. Nat Biotechnol 26: 702-708.

75. Berghmans S, Murphey RD, Wienholds E, Neuberg D, Kutok JL, et al. (2005) tp53 mutant zebrafish develop malignant peripheral nerve sheath tumors. Proc Natl Acad Sci U S A 102: 407-412.

76. Kim SH, Speirs CK, Solnica-Krezel L, Ess KC (2011) Zebrafish model of tuberous sclerosis complex reveals cell-autonomous and non-cell-autonomous functions of mutant tuberin. Dis Model Mech 4: 255-267.
This article was originally published in a special issue, Stem Cells-Cancer Research handled by Editor(s). Dr. Claudio Luparello, University of Palermo, Italy 